\title{
Composite magnetic anisotropy fabrics: experiments, numerical models, and implications for the quantification of rock fabrics
}

\author{
Bernard A. Housen, Carl Richter and Ben A. van der Pluijm \\ Department of Geological Sciences, University of Michigan, 1006 C.C. Little Building, Ann Arbor, MI 48109-1063, USA
}

(Received June 22, 1992; revised version accepted September 10, 1992)

\begin{abstract}
Housen, B.A., Richter, C. and van der Pluijm, B.A., 1993. Composite magnetic anisotropy fabrics: experiments, numerical models, and implications for the quantification of rock fabrics. Tectonophysics, 220: 1-12.

Magnetic fabrics from rocks with multiple mineral-preferred orientations may have anisotropy ellipsoids whose shape and orientation arise from the addition of two or more component fabrics. Our numerical models and experiments demonstrate that such composite magnetic fabrics do not directly reflect the shapes and/or orientations of the individual mineral fabrics, and we provide criteria for the recognition and interpretation of composite fabrics in natural rocks. These criteria include: (1) the orientation of the maximum susceptibility axis is located at the intersection of two planar fabrics, and (2) the shape of the susceptibility ellipsoid changes from oblate to prolate and the degree of anisotropy decreases, as the relative intensity of two planar component fabrics becomes equal and as the angle between the planar fabrics increases.

Composite magnetic fabrics are observed in the shales and slates of the Martinsburg Formation, Lehigh Gap, Pennsylvania. Modeling of the AMS (anisotropy of magnetic susceptibility) and ARMA (anhysteretic remanent magnetization anisotropy) behavior constrains the relative degree of anisotropy of the bedding-parallel and cleavage-parallel fabrics. In particular, ARMA model results allow a good estimate of magnetite fabric strength.

We conclude that, in the presence of composite magnetic fabrics, quantitative measures of finite strain in deformed rocks are limited by the ability to accurately determine the degree of anisotropy and relative susceptibility of each component fabric. Such determinations require knowledge of the mineral(s) that are responsible for the measured magnetic fabric and their behavior during deformation.
\end{abstract}

\section{Introduction}

Magnetic anisotropy fabrics measure the gross preferred orientation of either all minerals (AMS, anisotropy of magnetic susceptibility) or of magnetite (ARMA, anhysteretic remanent magnetization anisotropy), that are present in a rock sample. Magnetic fabrics can thus be used to quantify bulk rock fabrics (see reviews by Hrouda, 1982; Borradaile, 1988), and in some cases provide a measure of finite strain (review by Borradaile, 1991). The use of magnetic fabrics in structural geology, particularly for the analysis of strain, is

Correspondence to: B.A. Housen, Department of Geological Sciences, University of Michigan, 1006 C.C. Little Building, Ann Arbor, MI 48109-1063 USA. complicated by (1) compositional control of magnetic anisotropy variations (Borradaile, 1987), and (2) the effects of multiple mineral-preferred orientations (Borradaile and Tarling, 1981; Hrouda, 1992). Compositional control of magnetic fabric variations has been studied in recent years (Rochette and Vialon, 1984; Borradaile, 1987; Rochette, 1987; Borradaile and Sarvas, 1990; Housen and Van der Pluijm, 1990; Jackson, 1991) and will not be given further consideration in this paper. We will instead focus on the effect of multiple mineral-preferred orientations on magnetic anisotropy.

Multiple preferred-orientation fabrics are common in deformed rocks; a typical example is found in cleaved sediments, in which beddingparallel minerals define one planar fabric and cleavage-parallel minerals another planar fabric. 
Other examples of multiple-mineral fabrics include rocks with crenulation cleavage (S1 and S2), and S-C-type mylonites. When minerals that contribute significantly to magnetic anisotropy are present in more than one preferred orientation, the measured magnetic anisotropy will rcflect a composite of these orientation fabrics (Borradaile and Tarling, 1981). Such composite fabrics are likely to occur in many deformed rocks, and must be identified and compensated for when using magnetic fabric analysis to quantify rock fabrics, particularly when magnetic anisotropy is used as a measure of strain.

To examine the effects of multiple-mineral fabrics, we have conducted experiments with synthetic samples and numerically simulated composite magnetic fabrics. We demonstrate the applicability of our findings in a quantitative analysis of a natural composite fabric that is found in the Martinsburg Formation at Lehigh Gap, Pennsylvania.

\section{Experiments}

To test the effect of differing mineral-preferred orientations on magnetic anisotropy fabrics, we designed two experiments with synthetic samples. First, we examined the effect of relative variations in the susceptibility of two orthogonal fabrics, second we focused on the effect of variation in the angle between two planar fabrics. For both experiments, epoxy-magnetite discs were used for the planar fabrics. The discs were produced by mixing portions of $0.1-\mathrm{mm}$ spherical magnetite grains in diamagnetic epoxy poured into a mold. The discs had uniform dimensions of $2.5-\mathrm{cm}$ diameter and $0.2-\mathrm{cm}$ thickness. The amount of magnetite in the discs was varied in $0.02-\mathrm{g}$ increments from $0.02 \mathrm{~g}$ to $0.2 \mathrm{~g}$ (with $0.01 \mathrm{~g}$ increments from $0.08 \mathrm{~g}$ to $0.12 \mathrm{~g}$ ). The magnetic fabric of each disc was measured using an SI-2 AMS device. The susceptibility of each disc was linearly proportional to the amount of magnetite in the disc (Fig. 1), and ranged from $1.1 \times 10^{-3}$ to $11 \times 10^{-3}$ (SI volume susceptibility units are used throughout the paper). The discs were numbered according to the percentage of magnetite in the disc $(100 \%=0.2 \mathrm{~g})$. All the discs have oblate

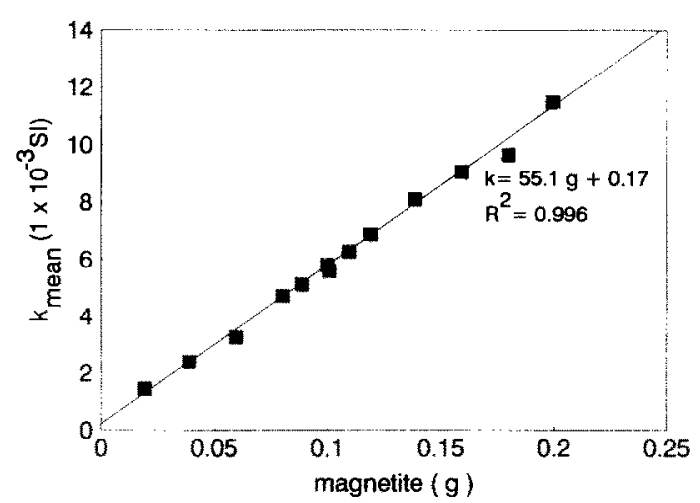

Fig. 1. Plot of the mean susceptibility $\left(1 \times 10^{-3}\right.$ SI volume units) of epoxy magnetite discs versus the weight of magnetite $(\mathrm{g})$. Correlation line and coefficient are also shown.

AMS ellipsoids (Fig. 2). The shape of the susceptibility ellipsoid is controlled by the shape of the discs, while the degree of anisotropy $(P=$ $k_{\text {max }} / k_{\text {min }}$, with $k_{\text {max }} \geqslant k_{\text {int }} \geqslant k_{\text {min }}$ as the principal susceptibility axes) is controlled by the amount of magnetite in each disc (see Appendix). Because the amount of magnetite controls the degree of anisotropy of the discs, the degree of anisotropy decreases from $P=1.28$ to $P=1.07$ with decreasing magnetite content. The orientation of the three principal susceptibility axes reflects the disc geometry, with the maximum and intermediate axes oriented in the plane of the disc, and the minimum axis perpendicular to the disc (Fig. 3a).

For our experiments, two of the epoxy-magnetite discs were placed in a diamagnetic clay matrix in a standard sample holder. The measured magnetic fabric is a composite fabric result-

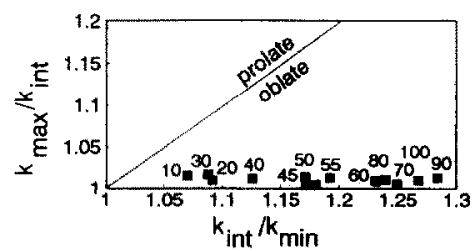

Fig. 2. Flinn-type diagram showing the AMS ellipsoid shapes of the individual discs. The vertical axis represents magnetic lineation $\left(k_{\max } / k_{\mathrm{int}}\right)$, the horizontal axis magnetic foliation $\left(k_{\text {int }} / k_{\min }\right)$. The numbers near the markers are the percentage of magnetite in the discs $(100=0.2 \mathrm{~g}, 90=0.18 \mathrm{~g}, 80=$ $0.16 \mathrm{~g}$, etc.). All discs have oblate ellipsnid shapes, with a degree of anisotropy which decreases with decreasing magnetite content (see Appendix). 
$\mathbf{a}$

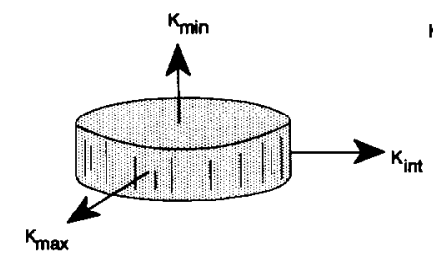

b

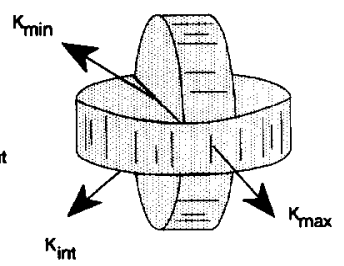

Fig. 3. Schematic illustration of the susceptibility axes of the discs and the composite disc fabrics. (a) The AMS axes of a single epoxy-magnetite disc, with the minimum axis perpendicular to the disc and the intermediate and maximum axes in the disc plane. (b) The AMS axes of a composite fabric arising from the addition of the susceptibilities of two orthogonal discs.

ing from the addition of the individual magnetic fabrics of each disc, which is shown schematically in Figure 3b.

\section{Experiment 1: Orthogonal fabrics}

The first experiment examines the effect of variation in the relative susceptibility and degree of anisotropy of two orthogonal discs. For this cxperiment the total susceptibility of the two discs is held constant at $11 \times 10^{-3}$. The susceptibility of each individual disc is varied: disc 1 defines the primary fabric which decreases in susceptibility from $9.9 \times 10^{-3}$ to $5.6 \times 10^{-3}$ and anisotropy from $P=1.28$ to $P=1.17$, and disc 2 defines the secondary fabric which simultaneously increases in susceptibility from $1.1 \times 10^{-3}$ to $5.7 \times 10^{-3}$ and in anisotropy from $P=1.07$ to $P=1.17$. Disc 1 is horizontal in the sample holder, while disc 2 is vertical, with an E-W strike (in sample coordinates). The results of this experiment are shown in Figure 4. The shape of the susceptibility ellipsoid trends from oblate to prolate as the susceptibilities of the two fabrics approach equality (disc $1=5.6 \times \times 10^{-3}, P=1.17$; disc $2=5.7 \times 10^{-3}$, $P=1.17$; Fig. 4a). The susceptibility axes (Fig. 4b) remain coaxial with disc $1\left(k_{\max }, k_{\text {int }}\right.$ parallel to the plane of the disc, $k_{\min }$ perpendicular to the disc), until disc 2 reaches $>30 \%$ of the total susceptibility. As the two discs approach equal susceptibilities, the minimum and intermediate axes shift about $10^{\circ}$. The maximum axes remain fixed at the line of intersection of the two discs a
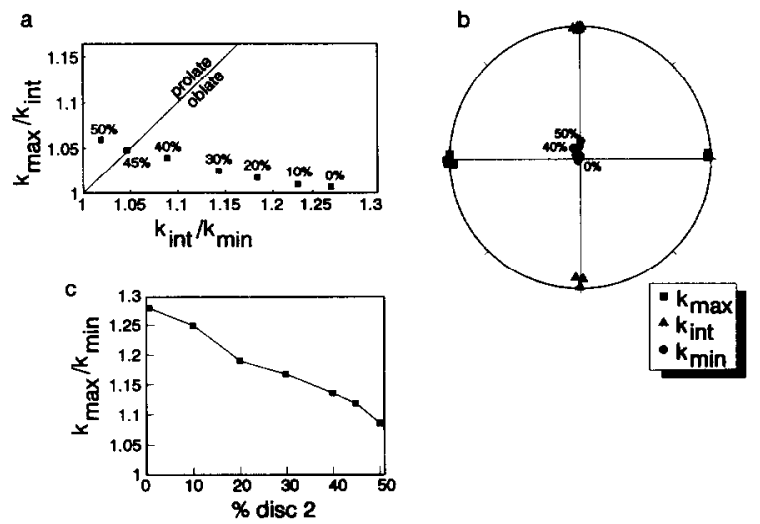

Fig. 4. Results of experiment 1; two orthogonal, oblate fabrics. (a) Flinn-type diagram showing the effect of variations in the relative susceptibility (magnetite content) of two orthogonal, oblate epoxy-magnetite discs. The numbers denote the relative susceptibility of the weaker disc, the total susceptibility of the sample is constant. As the two discs become equal in susceptibility, the shape of the measured AMS ellipsoid trends from oblate to prolate. (b) Equal-area, lower-hemisphere projection of the principal susceptibility axes $\left(k_{\max }, k_{\text {int }}, k_{\min }\right)$. The disc with the higher susceptibility is horizontal, the other disc is vertical with an E-W strike (in sample coordinates). The numbers shown in the projection correspond to the relative intensity of the weaker disc for three experimental steps. The AMS axes parallel the shape of the stronger disc when the weaker disc is less than $40 \%$ of the total susceptibility, as the two discs approach equal susceptibilities the minimum and intermediate AMS axes shift about $10^{\circ}$. The maximum axes are always parallel to the intersection line between the two discs. (c) Degree of anisotropy $\left(P=k_{\max } / k_{\min }\right)$ versus the relative susceptibility of disc 2 (the initially weaker fabric). As the two fabrics become equal in susceptibility, the degree of anisotropy decreases.

regardless of their relative susceptibilities or their degree of anisotropy. The degree of anisotropy of the composite fabric decreases from $P=1.28$ to $P=1.10$ (Fig. 4c) as the two component fabrics become equal in susceptibility and anisotropy.

\section{Experiment 2: Non-orthogonal fabrics}

The second experiment examines the effect of variations in the angle between two oblate magnetic fabrics. We use two discs of equal susceptibility, with a uniaxial-oblate anisotropy. Both discs are initially vertical in the sample holder with an $\mathrm{E}-\mathrm{W}$ strike, one disc remains in this orientation, while the other is rotated to a horizontal orientation in $15^{\circ}$ increments. The shape of the suscepti- 
a

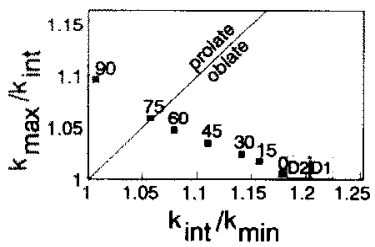

c

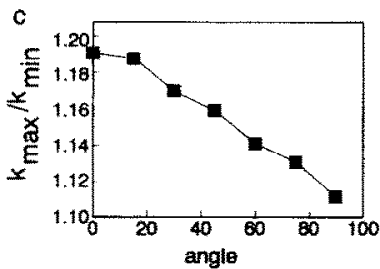

Fig. 5. Results of experiment 2: varying angles between two oblate fabrics. (a) Flinn-type diagram showing the effect of changes in the angle between two epoxy-magnetite discs on AMS ellipsoid shape. The two discs (DI and $D 2)$ are essentially equal in both susceptibility and degree of anisotropy. The number near the marker is the angle between the two discs. As the angle increases, the AMS ellipsoid changes non-linearly from oblate to prolate shapes. (b) Equal-area, lower-hemisphere projection of the principal AMS axes. The two discs are both initially parallel and vertical with an East-West strike. The rotation angle gives the rotating disc a southerly dip. The angle between the discs for four steps is shown for reference. The minimum and intermediate AMS axes change orientation as a function of the angle between the discs. The maximum axes remain at the intersection line between the two discs. (c) Degree of anisotropy versus the angle between the two planar discs. The degree of anisotropy decreases from 1.19 to 1.09 as the angle between the two discs increases.

bility ellipsoid varies nonlinearly with the rotation angle, trending from oblate to prolate as the angle between the discs increases (Fig. 5a). The prolate field is reached at an angle of ca. $75^{\circ}$. The minimum and intermediate susceptibility axes bisect the angle between the two discs (Fig. 5b). Again, the maximum axes remains fixed at the line of intersection between the two discs throughout the experiment. The degree of anisotropy of the composite fabric decreased from $P=1.19$ to $P=1.11$ as the angle between the discs increased from $0^{\circ}$ to $90^{\circ}$ (Fig. $5 \mathrm{c}$ ).

\section{Numerical modeling}

More complex experiments are relatively difficult to perform with synthetic samples. Numerical modeling, however, provides an easy method to examine composite fabrics with more variable components. To test this approach, we first compare numerical simulations with our experimental results. Our numerical model utilizes the tensor properties of magnetic fabrics to characterize the total and component fabrics. The total magnetic fabric can be described by a second-order tensor $\left[k_{i j}\right]$ which is the sum of two (or more) secondorder tensors that describe the orientation and magnitude of the individual fabrics. In our calculations the individual component fabrics are perfectly uniaxial-oblate $\left(k_{\max }=k_{\text {int }}>k_{\min }\right)$, and are defined by the polar coordinates of the minimum axis and the magnitudes of the maximum and minimum axes. The equations which are used to calculate the six independent components of the fabric tensor are given in Owens (1974). Summation of these components yields the composite fabric tensor and solving for the eigenvalues and the eigenvectors of this tensor yields the magnitudes and orientations of the principal susceptibility axes of the composite magnetic fabric.

\section{Model 1}

The first numerical model uses parameters similar to our first experiment. The two orthogonal fabrics are oblate with a degree of anisotropy which varies from $P=1.28$ to $P=1.07$. The total susceptibility of the simulated sample is held constant while the relative intensity of the two component fabrics is varied, with the initially-stronger fabric decreasing in magnitude (from $100 \%$ to $50 \%$ of the total), and the initially-weaker fabric increasing in magnitude (from $0 \%$ to $50 \%$ ). The degree of anisotropy of each fabric was varied with the fabric susceptibility to accurately simulate the first experiment, with the initially-stronger fabric decreasing in anisotropy from $P=1.28$ to $P=1.17$, and the initially-wcakcr fabric increasing from $P=1.07$ to $P=1.17$. The initiallystronger fabric is oriented horizontally, the other fabric has a vertical dip and an east-west strike. The shape of the model ellipsoid trends from oblate to prolate as the two fabrics become equal in susceptibility and anisotropy (Fig. 6a). The minimum axis is orthogonal to the horizontal fabric, and shifts $5^{\circ}$ when the susceptibility of the 

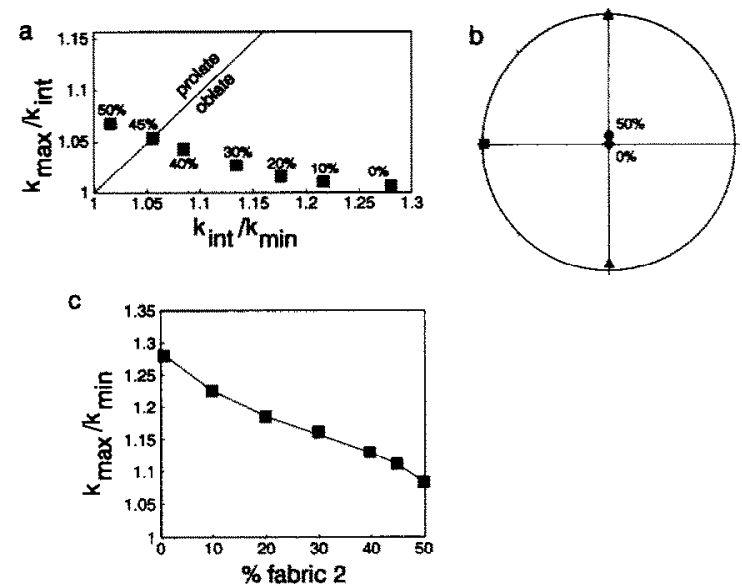

Fig. 6. Numerical model for two orthogonal oblate fabrics with variable relative susceptibility and degree of individual fabric anisotropy. The susceptibility and anisotropy of one fabric increases as the second fabric decreases. The bulk susceptibility is held constant. The initially-stronger fabric is horizontal, the other vertical with a E-W strike. (a) Flinn-type diagram showing changes in ellipsoid shape as the relative magnitudes change. Note the trend from oblate to prolate shapes. The numbers denote the relative magnitude of the weaker fabric. (b) Changes in the orientation of the principal axes as the relative susceptibilities of the component fabrics change. The numbers denote the vertical fabric susceptibility $(0-50 \%)$. The axes are parallel to the shape of the stronger fabric. The maximum axes are always parallel to the intersection of the two planar fabrics. (c) Degree of anisotropy ( $P=$ $k_{\max } / k_{\min }$ ) versus the relative susceptibility of fabric 2 (the initially weaker fabric). As the two fabrics become equal in susceptibility, the total degree of anisotropy decreases.

fabrics is the same (Fig. 6b). A similar trend is observed for the intermediate axis, while the maximum axis remains fixed at the line of intersection of the two fabrics throughout the simulation. The degree of anisotropy of the composite fabric decreases from the initial value of $P=1.28$ to a value of $P=1.09$ when the two fabrics are of equal susceptibility and anisotropy (Fig. 6c). These trends are essentially identical to those found in our first experiment with the epoxy-magnetite discs.

\section{Model 2}

The second numerical simulation mimics the second experiment, in which the angle between two planar fabrics is varied. Two fabrics with equal susceptibilities and equal anisotropies $(P=$
1.2) are initially parallel. The orientation of fabric 1 is fixed, fabric 2 is rotated in $10^{\circ}$ increments until it is orthogonal to fabric 1 . The flexibility of the numerical model allows the use of a smaller rotation angle than in the experiments, so the variations in the composite fabrics with angle can be examined in greater detail. Figure 7 shows the results of this simulation. The shape of the composite ellipsoid changes nonlinearly from oblate to prolate as the angle between the two component fabrics increases (Fig. 7a). The minimum and intermediate axes of the composite ellipsoid have orientations in between the two individual fabrics; these orientations change systematically as the angle between the two fabrics increases (Fig. 7b). The maximum axis remains at the intersection between the two fabrics. The degree of anisotropy decreases from $P=1.2$, when the two fabrics are parallel, to $P=1.1$ when the fabrics are orthogonal (Fig. 7c). These results are essen-
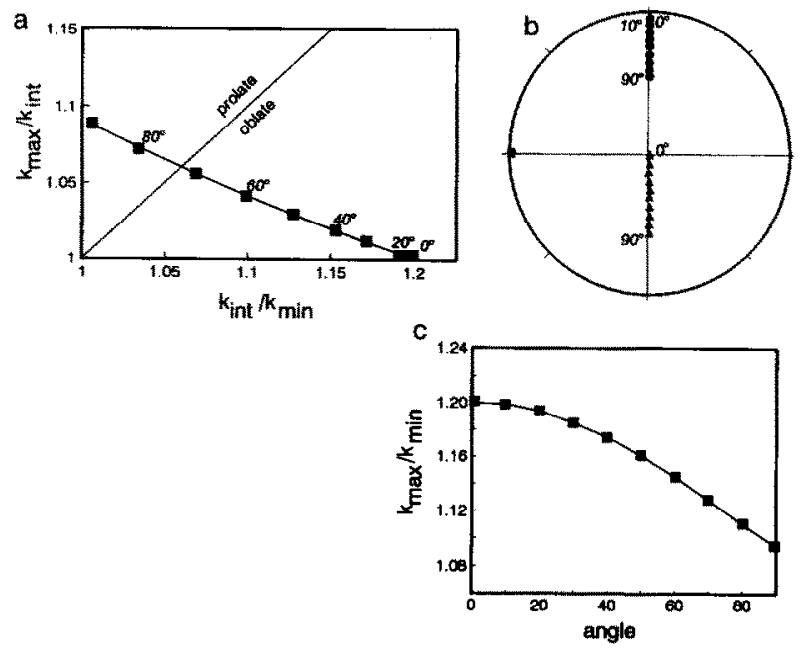

Fig. 7. Results of numerical model with changing angle between two constant susceptibility, constant anisotropy ( $P=$ 1.2), oblate fabrics. (a) Changes in ellipsoid shape of the composite magnetic fabric with changing angle between the two fabrics. Note the non-linear progression from oblate to prolate shapes as the angle increases. (b) Changes in orientation of the principal susceptibility axes with changes in the angle between the two fabrics. The minimum and intermediate axes have orientation in between the two fabrics, and rotate with increasing angle between the fabrics. The maximum axes remain at the intersection of the two fabrics. (c) Degree of anisotropy versus the angle between the two planar fabrics. The degree of anisotropy decreases from 1.2 to 1.08 as the angle between the two fabrics increases. 

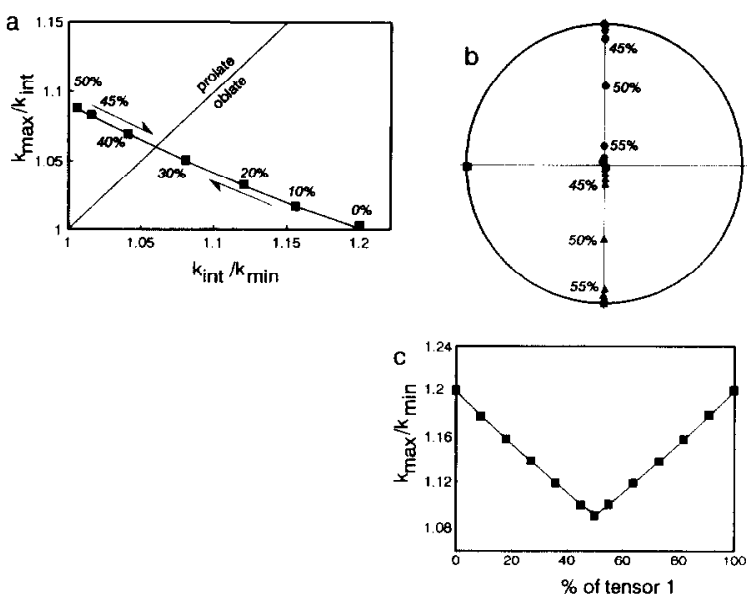

Fig. 8. Numerical model for two orthogonal uniaxial-oblate fabrics with variable relative susceptibility. The susceptibility of one fabric increases as the second fabric decreases. The bulk susceptibility and degree of individual fabric anisotropy $(P=1.2)$ is held constant. The initially-stronger fabric is vertical with a E-W strike, the other is horizontal. (a) Flinn-type diagram showing changes in ellipsoid shape as the relative magnitudes change. Note the trend from oblate to prolate back to oblate shapes. The numbers denote the relative magnitude of the weaker fabric. (b) Changes in the orientation of the principal axes as the relative susceptibilities of the component fabrics change. The numbers denote the horizontal fabric susceptibility $(45-55 \%)$ for intermediate orientations. The axes are parallel to the stronger fabric when the difference in fabric susceptibilities is large. Intermediate orientations occur when the fabrics have similar susceptibilities. The maximum axes are always parallel to the intersection of the two planar fabrics. (c) Degree of anisotropy $\left(P=k_{\max } / k_{\min }\right)$ versus the relative susceptibility of tensor 1 (the initially weaker fabric). As the two fabrics become equal in susceptibility, the total degree of anisotropy decreases.

tially identical to those of our second experiment (compare with Fig. 5).

\section{Model 3}

The third model simulates composite fabrics arising from two uniaxial-oblate fabrics with a constant degree of anisotropy $(P=1.2)$. This simulation models the effect of variations in two orthogonal mica fabrics on the total magnetic fabric. The total susceptibility of the simulated sample is held constant while the relative susceptibility of the two component fabrics is varied, with the initially-stronger fabric decreasing in magnitude (from $100 \%$ to $0 \%$ of the total), and the initially-weaker fabric increasing in magnitude (from $0 \%$ to $100 \%$ ). The initially-stronger fabric is oriented with a vertical dip and an eastwest strike, the other fabric is horizontal. The shape of the model ellipsoid trends from oblate to prolate as the two fabrics become equal in susceptibility (Fig. 8a). The minimum axis is always orthogonal to the stronger of the two fabrics when the difference in the susceptibility of the fabrics is large (Fig. 8b). When the two fabrics are nearly equal in susceptibility (between $45 \%$ and $55 \%$ ), the minimum axis is oriented in between the two fabrics. A similar trend is observed for the intermediate axis, while the maximum axis remains fixed at the line of intersection of the
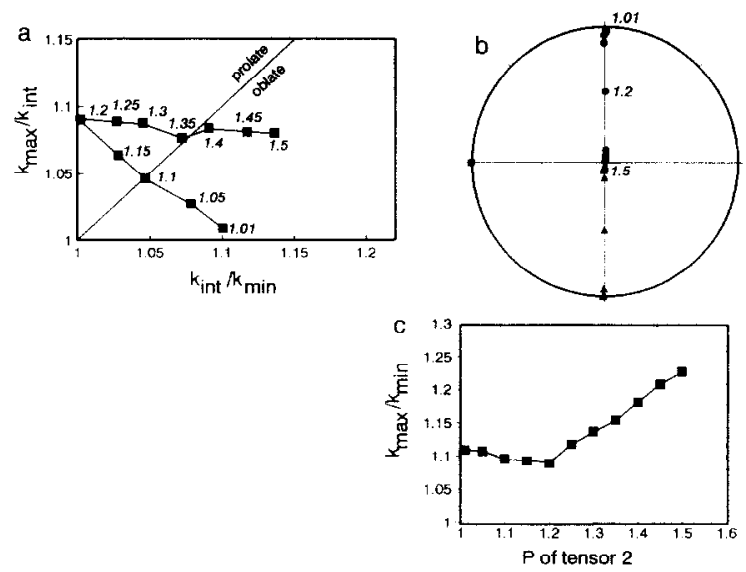

Fig. 9. Results of numerical model with one fabric held at constant degree of anisotropy $(P=1.2)$, and the other fabric increasing in anisotropy from $P=1.01$ to $P=1.5$. Both fabrics are maintained at constant susceptibility, and the two fabrics have orthogonal orientations. The constant anisotropy fabric is vertical with an E-W strike, the other fabric is horizontal. (a) Flinn-type diagram of the fabric development. The numbers in the plot give the degree of anisotropy of the variable fabric. As $P$ of the variable fabric increases from 1.01 to 1.2 , the composite ellipsoid shape trends from oblate to prolate shapes. As $P$ increases further to 1.5 , the composite fabric becomes triaxially-oblate. (b) Changes in principal axes orientations. The axes parallel the most-anisotropic fabric. When the two fabrics are of equal anisotropy, the minimum and intermediate axes lie in between the two fabrics. The maximum axes are always parallel to the intersection of the two fabrics. (c) Degree of anisotropy of the composite fabric versus the degree of anisotropy of the variable fabric (tensor 2). The degree of anisotropy of the composite fabric changes very little as the anisotropy of the variable fabric increases from 1.01 to 1.2 , then increases as the variable anisotropy increases to 1.5 . 
two fabrics throughout the simulation. The degree of anisotropy of the composite fabric decreases from the initial value of $P=1.2$ to a value of $P=1.09$ when the two fabrics are of equal susceptibility (Fig. 8c).

\section{Model 4}

A more complex example in which a fabric increases its degree of anisotropy is examined in the fourth numerical simulation. Two orthogonal fabrics are used, fabric 1 with a constant $(P=1.2)$ degree of anisotropy, fabric 2 with an anisotropy which increases from $P=1.01$ to $P=1.5$. The bulk susceptibility of both fabrics is equal. The shape of the composite susceptibility ellipsoid trends from uniaxial-oblate when fabric 2 has $P=1.01$ (oblate when $P<1.1$ ), to prolate when $1.35>P>1.1$ (uniaxial-prolate when $P=1.2$ ) (Fig. 9a). The anisotropy evolves to an oblate shape when the anisotropy of fabric 2 is $>1.35$. The minimum axes of the composite fabrics are perpendicular to the component fabric with the higher degree of anisotropy (Fig. 9b), and have an intermediate orientation when the AMS of both fabrics is equal. The degree of anisotropy of the composite fabric remains relatively constant as fabric 2 increases in anisotropy to $P=1.2$. After this fabric 2 dominates and the resulting composite AMS increases as the anisotropy of the variable fabric 2 increases to $P=1.5$ (Fig. 9c).

\section{Identification of composite fabrics}

Using the results from our experiments and numerical modeling, we can develop some criteria to identify and interpret composite fabrics in natural rocks. We limit our discussion to commonly observed composite fabrics arising from two planar rock fabrics, such as bedding and cleavage.

The one characteristic common to all composite fabrics is that the maximum susceptibility axis is always oriented at the intersection of two planar fabrics. The orientations of the minimum and intermediate susceptibility axes are relatively insensitive to the effects of multiple mineral fabrics when one of the fabrics dominates by either larger bulk susceptibility or greater degree of anisotropy. Minimum and intermediate axis orientations in between the two fabrics are only expected when the fabrics are very similar in susceptibility and degree of anisotropy. Thus the minimum and intermediate axes can serve as composite fabric indicators only when the two component fabrics contribute equally to the total magnetic anisotropy. The maximum axis orientation is a reliable indicator of composite fabrics.

Changes in the shape and degree of anisotropy of the susceptibility ellipsoid as a function of (1) the angle between planar fabrics, or (2) the relative intensity of the component fabrics, can be used to identify and interpret composite fabrics. For variable angles, our results show that as the angle between two planar fabrics increases, the degree of anisotropy decreases and the shape of the susceptibility ellipsoid trends from an oblate to a prolate shape. Such trends will occur in rocks where the angle between two planar fabrics varies; for example, with cleavage across a fold. When the relative intensity of two planar fabrics varies, our results show that as two planar fabrics become equal in susceptibility and degree of anisotropy, the shape of the composite susceptibility ellipsoid trends from oblate to prolate shapes, and the degree of anisotropy decreases. This variation is also a function of the angle between the two fabrics, and is less pronounced when the angle between the fabrics is small. Observation of similar variations in the shape and anisotropy of the susceptibility ellipsoid where the relative strength of two planar fabrics varies in natural rocks, such as progressive development of slaty cleavage or of mylonitic foliation, indicates that the measured anisotropy represents a composite magnetic fabric.

\section{An application}

The magnetic anisotropy (both AMS and ARMA) results from the shale to slate transition in the Martinsburg Formation at Lehigh Gap, Pennsylvania, provide an opportunity to examine the effects of composite magnetic fabrics. The geometry of these composite fabrics also provides information on the behavior of the associated 
minerals in these rocks during cleavage formation.

The Martinsburg Formation at Lehigh Gap, Pennsylvania, is an example of progressive slaty cleavage development. A change from uncleaved shale to well-cleaved slate is preserved in a strain shadow beneath the overlying, more competent Shawangunk Formation (Epstein and Epstein, 1969). The degree of cleavage development is proportional to the distance from the Martinsburg-Shawangunk contact, with uncleaved shales occurring 0-70 m from the contact, pencil slates $70-100 \mathrm{~m}$, and well-cleaved slates $100 \mathrm{~m}$ and beyond. Microstructural and X-ray texture studies (Holeywell and Tullis, 1975; Wintsch, 1978; Lee et al., 1986) have shown that chlorite and mica are sub-parallel to bedding in the shales and cleavage-parallel in the slates, and that the phyllosilicates mainly reorient via dissolution and new-growth during cleavage development. The relative proportion of bedding-parallel phyllosilicates decreases, and the relative proportion of cleavage-parallel phyllosilicates increases as cleavage develops. Field measurements show that the two mineral-preferred orientations present in these rocks are orthogonal.

\section{AMS fabrics}

Anisotropy of magnetic susceptibility (AMS) study of these rocks (Housen and van der Pluijm, $1990,1991)$ found that the AMS fabrics are controlled by chlorite. The orientation of the maximum susceptibility axes for all samples is parallel to the bedding-cleavage intersection, the minimum axes are nearly-perpendicular to bedding in the shale and pencil slates, and perpendicular to cleavage in the well-cleaved slates. No orientations of the axes in between bedding and cleavage are observed. The shape of the AMS ellipsoid (Fig. 10) trends from oblate shapes in the shales to the oblate/prolate boundary in the slates. The trend in susceptibility ellipsoid shapes is similar to that observed in our models of composite fabrics resulting from the progressive addition of two oblate fabrics with one fabric decreasing, the other increasing in susceptibility (Fig. 8). This is consistent with the observed behavior of chlorite

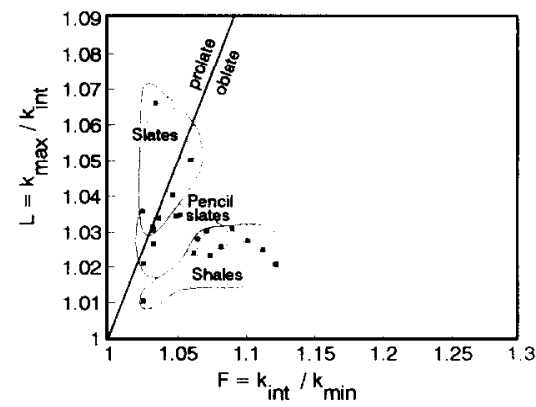

Fig. 10. Flinn-type diagram showing the shape of the AMS ellipsoids from the shale-slate transition in the Martinsburg Formation at Lehigh Gap. The shape trends from oblate for the end-member shale, to prolate for the well-cleaved slate.

Results from Housen and Van der Pluijm (1990, 1991).

in these rocks, and indicates that the measured magnetic fabric in the slates is a composite fabric. One of the end-member magnetic fabrics (the shale fabric) is known, so numerical modeling can be applied to determine the relative proportions of bedding-parallel versus cleavage-parallel chlorite across the shale to slate transition, using the measured composite fabric and the initial shale fabric. First, however, we must constrain the possible values for the anisotropy of the slate fabric. Since the AMS is controlled by chlorite, the shape and degree of anisotropy of the slate fabric is limited by the single-mineral anisotropy of chlorite. For the Fe-rich chlorite found in the Martinsburg Formation (Wintsch et al., 1991) the single-mineral anisotropy is $P=1.2-1.3$ (Borradaile et al., 1987), and is uniaxial-oblate. This anisotropy represents the upper limit of the anisotropy of cleavage-parallel chlorite with a perfect preferred orientation $\left(P_{\max }\right)$; if the cleavage-parallel chlorite is not perfectly oriented, the degree of anisotropy of the cleavage fabric will be lower than $P_{\max }$. The measured bulk susceptibility of the samples from the shale-slate transition is constant, which indicates that the total amount of chlorite in these rocks remains relatively constant. The combined component fabrics in the model must, therefore, maintain a constant volume of chlorite.

To model the AMS fabrics in these rocks we use the initial shale fabric, the constant bulk susceptibility of the composite fabric, and oblate component fabrics that are orthogonal. Two slate 
fabrics are illustrated, one with $P=1.2-1.3$ (representing perfectly-aligned chlorite) and another with $P=1.1$ (representing chlorite with a lower degree of preferred orientation). The development of the cleavage via dissolution/new-growth of chlorite is modeled by increasing the relativc susceptibility of the cleavage-parallel fabric from $0-100 \%$ of the total, while simultaneously decreasing the bedding-parallel fabric from $100-0 \%$. Figure 11a shows the results of our modeling using a cleavage fabric with $P=1.2-1.3$ (ruled area), and results for the cleavage fabric with $P=1.1$ (solid line). The numbers on the trends are the percentage of the cleavage fabric susceptibility relative to the total susceptibility. The initial shale fabric is represented by the solid oval ( $L=1.02, F=1.12$ ). Shown by solid squares are the AMS results from the Martinsburg Formation. The progression from the oblate initial fabric, to prolate shapes, back to the oblate endmember, matches the general trend observed in the Martinsburg Formation AMS. The prolate AMS cllipsoid shapes in the well-cleaved Martinsburg slates are only attained using $P=1.2-1.3$ for the cleavage-parallel fabric in the model. Lower $P$ values do not produce the prolate slate fabric that is observed. The ellipsoid shapes obtained from the model using $P=1.2-1.3$ do not, however, pass through the measured AMS ellipsoids of the pencil slates. These AMS ellipsoid shapes are only obtained by using lower anisotropies $(P=1.01-1.10)$ for the initially-developed slate fabrics in the numerical model. Figure $11 \mathrm{~b}$ shows the model results using $P=$ 1.01-1.10 for the initial slate fabric $(0-30 \%$ of the total susceptibility), and $P=1.2-1.3$ for the remainder $(30-100 \%)$ of the slate fabric, with the AMS results also plotted. The fit remains limited, but the modeling does suggest that the cleavageparallel chlorite which formed during the incipient stages of cleavage formation had a relatively low degree of preferred orientation, and that chlorite which formed during the later stages of cleavage development had a high degree of preferred orientation. Using Figure 11, we estimate that $<20 \%$ of the chlorite is parallel to the incipient cleavage, $<30 \%$ of the chlorite is cleavage-parallel in the pencil slates, and $>30 \%$ of the chlorite is cleavage-parallel in the slates. Because the slates farthest from the MartinsburgShawangunk contact have prolate AMS ellip-

a

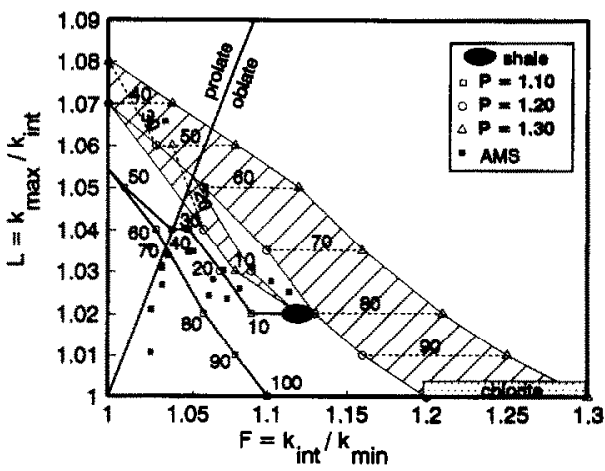

b

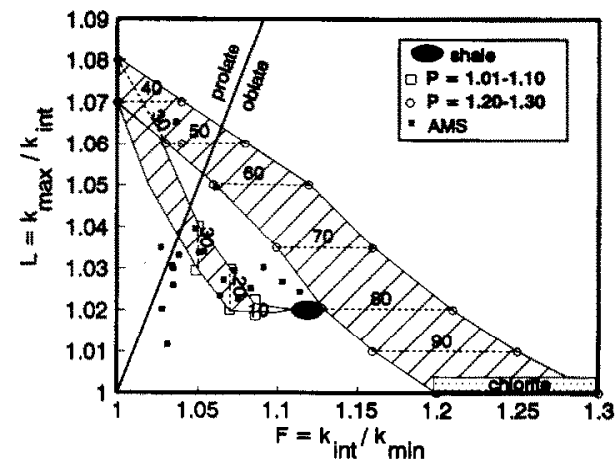

Fig. 11. Numerical model of composite AMS fabrics. (a) Using the initial shale AMS and slate fabrics with $P=1.1$ and $P=1.2-1.3$. The latter $P$ values are the single mineral anisotropy values for $\mathrm{Fe}$-rich chlorite. A constant total susceptibility is maintained by the model, with the relative susceptibility of the shale fabric decreasing from 100 to $0 \%$, and the relative susceptibility of the slate fabric increasing from 0 to $100 \%$. The numbers on each trend refer to the relative susceptibility of the slate fabric for each model step. The initial shale fabric is plotted as an oval. The measured AMS ellipsoid shapes are also plotted. The general trend from the oblate, initial shale fabric to prolate composite ellipsoids matches the general trend observed in the Martinsburg AMS results. The AMS ellipsoids of the well-cleaved slates matches the numerical fabric with $P=1.2-1.3$. The measured pencil slate AMS ellipsoids have a lower degree of anisotropy than the modeled composite ellipsoids with $P=1.2-1.3$, which suggests the initially-formed cleavage parallel chlorite has a relatively low degree of anisotropy. (b) Numerical model of composite AMS fabrics using $P=1.01-1.1$ for the initial (0-20\% of total) cleavage-parallel fabric, and $P=1.2-1.3$ for the remainder $(30.100 \%)$ of the cleavage-parallel fabric Compare the model results with the Martinsburg AMS ellipsoid shapes. 
soids, bedding-parallel chlorite must contribute significantly to the composite fabric of these slates. The AMS of these slates is, therefore, not an accurate measure of the preferred-orientation of the cleavage-parallel chlorite, and the AMS ellipsoid from these samples cannot provide even a qualitative estimate of the strain magnitudes in these rocks.

\section{ARMA fabrics}

Study of the ARM anisotropy (ARMA) found that the shape of the ARMA ellipsoid (which measures the preferred orientation of magnetite) trends from oblate, bedding-parallel ellipsoids in the shale, to slightly prolate shapes in the pencil slates, to oblate, cleavage-parallel shapes in the slates (Fig. 12a; Housen and Van der Pluijm, 1991). For most of the shales, all the pencil slates, and most of the well-cleaved slates, the orientation of the maximum ARMA axes is parallel to the bedding-cleavage intersection. Orientations of the minimum ARMA axes in between the bedding and cleavage orientations, similar to those observed in the models (Figs. 4 and 6), are observed in the pencil slate samples. These observations all indicate that the ARMA is a composite magnetic fabric that arises from two orthogonal, magnetite preferred-orientations, one bedding parallel, the other cleavage parallel. Since the ARM intensities are relatively constant, a constant volume of magnetite is assumed. To maintain a constant total volume of magnetite the bedding-parallel magnetite must dissolve and grow parallel to cleavage as the slaty cleavage develops (Housen and van der Pluijm, 1991).

We estimate the relative proportions of bedding-parallel magnetite and cleavage-parallel magnetite by numerical modeling. Using the orientation and anisotropy of the initial shale fabric and the final cleavage fabric as end-members, we decrease the relative ARM intensity of the shale fabric from $100 \%$ to $0 \%$ of the total, while simultaneously increasing the relative ARM intensity of the slate fabric from $0 \%$ to $100 \%$, in accordance with our observations of magnetite behavior. The general trend of ellipsoid shapes produced by the model (Fig. 12b) is identical to that
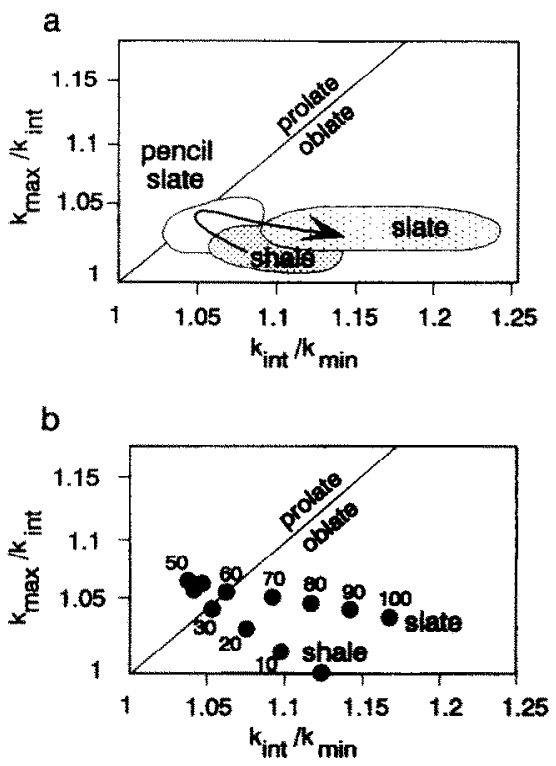

Fig. 12. Measured and numerical model ARMA fabrics of the Martinsburg Formation. (a) Summary Flinn-type diagram showing the measured ARMA ellipsoid shapes from the shale-slate transition. The shape of the ARMA ellipsoids trend from oblate shale, to slightly-prolate pencil state, to oblate slate with progressive cleavage formation. (Results from Housen and Van der Pluijm, 1991), (b) Flinn-type diagram showing the ellipsoid shapes of the composite fabrics from the numerical model. The initial shale and final slate fabrics are indicated. The numbers marking the results are the percentage of the slate fabric relative to the total fabric. Note the trend towards the prolate-shape field with relatively small fractions of the slate fabric.

of the observed ARMA ellipsoids. Based on this we estimate that $<20 \%$ of the magnetite is parallel to the incipient cleavage in the shales, 20$70 \%$ of the magnetite is parallel to cleavage in the pencil slates, and $>70 \%$ of the magnetite is parallel to cleavage in the well-cleaved slates.

\section{Conclusions}

The identification of composite magnetic fabrics is vital to the reliable application of magnetic anisotropy to structural problems, particularly when anisotropy is used as a measure of finite strain. Our numerical and experimental results show that the shape of the susceptibility ellipsoid is particularly sensitive to the effects of multiple mineral fabrics. We find that when composite 
fabrics are present not only the magnitude, but also the orientations of the susceptibility ellipsoid, may not correspond to the orientation and magnitude of the strain ellipsoid. Since the shape of the susceptibility ellipsoid is used in all magnetic fabric-strain corrclations (Borradaile, 1991), these correlations are also sensitive to the effects of composite magnetic fabrics. The observation of the maximum susceptibility axis oriented at the bedding-cleavage intersection in many magnetic anisotropy studies of slates (see review by Borradaile, 1988) would suggest that composite magnetic fabrics are very common in slates, and this may explain some of the difficulties in establishing AMS-strain correlations.

The important observation from the ARMA model is that variations in the relative amount of magnetite in each of the component fabrics can produce a significant variation in the shape of the ARMA ellipsoid. These composite fabric variations can produce changes in the magnetic fabric which are larger than those produced by an increasing degree of mineral-preferred orientation during deformation. Because the shape of the susceptibility ellipsoid is used to quantify rock fabrics, identification of the effects of composite fabric variations is vital to any attempt to quantitatively separate deformation fabrics from total (composite) magnetic fabrics. In rocks where a gradual development of the component fabrics is not preserved, or in which significant compositional variations exist, it is difficult to accurately determine the contributions ofindividual fabrics to the composite magnetic fabric. Therefore, magnetic fabric-strain correlations based on tensor separation methods such as Hrouda (1992), should not be attempted unless the effects of both compositional variation and composite fabrics are considered.

\section{Acknowledgments}

This work was supported by NSF grant EAR9119196, and DFG grant RI 576/1-1. We thank Rob van der Voo and Jay Busch for their comments during our work, and Graham Borradaile and Mike Jackson for their useful reviews of this manuscript.

\section{Appendix}

The equation describing the susceptibility of the experimental epoxy-magnetite discs (modified from Piper, 1987):

$k_{i}=\frac{f_{\mathrm{mgt}}}{1 / k_{\mathrm{mgt}}+\left[\left(v_{\mathrm{d}} / v_{\mathrm{s}}\right) N_{\mathrm{mgt}}+f_{\mathrm{mgt}} \times N_{\mathrm{i}}\right]} \times\left(1 / v_{\mathrm{d}}\right)$

The measured volume-susceptibility axes $\left(k_{\mathrm{i}}\right)$ are a function of the single grain susceptibility $\left(k_{\mathrm{mgt}}\right)$ and demagnetization factor $\left(N_{\mathrm{mgt}}\right)$ of the magnetite grains in the discs, the shape anisotropy of the discs determined by the demagnetization factors $\left(N_{\mathrm{i}}\right)$ appropriate for the disc shape (after Pfleiderer and Halls, 1990), the volume of magnetite, the disc volume $\left(v_{\mathrm{d}}\right)$, and the sample volume $\left(v_{\mathrm{s}}\right)$. In the equation, the volume of magnetite is given as the volume fraction of magnetite $\left(f_{\mathrm{mgt}}\right)$ relative to the disc volume, $f_{\mathrm{mgt}}=$ vol. magnetite $/ v_{\mathrm{d}}$ (after Piper, 1987). The anisotropy of the discs is calculated by treating the discs as a single magnetite grain (Pfleiderer and Halls, 1990), so the demagnetization factor is a function of the disc shape. Because the contribution of the disc shape ( $N_{\mathrm{i}}$ in the equation) to the measured anisotropy is a function of the volumc-fraction of magnetite in the disc $\left(f_{\mathrm{mgt}}\right)$, the degree of anisotropy $\left(P=k_{\max } / k_{\min }\right)$ will decrease with decreasing magnetite content.

\section{References}

Borradaile, G.J., 1987. Anisotropy of magnetic susceptibility: rock composition versus strain. Tectonophysics, 138: 327329.

Borradaile, G.J., 1988. Magnetic susceptibility, petrofabrics and strain. Tectonophysics, 156: 1-20.

Borradaile, G.J., 1991. Correlation of strain with anisotropy of magnetic susceptibility (AMS). Pure Appl. Geophys., 135: 15-29.

Borradaile, G.J. and Sarvas, P., 1990. Magnetic susceptibility fabrics in slates: structural, mineralogical and lithological influences. Tectonophysics, 172: 215-222.

Borradaile, G.J. and Tarling, D.H., 1981. The influence of deformation mechanisms on magnetic fabrics in weakly deformed rocks. Tectonophysics, 77: 151-168.

Borradaile, G.J., Mothersill, J., Tarling, D. and Alford, C., 1986. Sources of magnetic susceptibility in a slate. Earth Planet. Sci. Lett., 76: 336-340.

Borradaile, G.J., Keeler, W., Alford, C. and Sarvas, P., 1987 Anisotropy of magnetic susceptibility of some metamorphic minerals. Phys. Earth Planet. Inter., 48: 161-166. 
Epstein, J.B. and Epstein, A.G., 1969. Geology of the Valley and Ridge province between Delaware Water Gap and Lehigh Gap, Pennsylvania. In: S. Subitzky (Editor), Geology of Selected Areas in New Jersey and Eastern Pennsylvania, and Guidebook of Excursions. Rutgers Univ. Press, New Brunswick, N.J., pp. 132-205.

Holeywell, R.C. and Tullis, T.E., 1975. Mineral reorientation and slaty cleavage in the Martinsburg Formation, Lehigh Gap, Pennsylvania. Geol. Soc. Am. Bull., 86: 1296-1304.

Housen, B.A. and van der Pluijm, B.A., 1990. Chlorite control of correlations between strain and anisotropy of magnetic susceptibility. Phys. Earth Planet. Inter., 61: 315-323.

Housen, B.A. and van der Pluijm, B.A., 1991. Slaty cleavage development and magnetic anisotropy fabrics. J. Geophys. Res, 96: 9937-9946.

Hrouda, F, 1982. Magnetic anisotropy of rocks and its application in geology and geophysics. Geophys. Surv, $5: 37-82$.

Hrouda, F., 1992. Separation of a component of tectonic deformation from a complex magnetic fabric. $\mathbf{J}$. Struct. Geol, 14: 65-71.

Jackson, M., 1991. Anisotropy of magnetic remanence: a brief review of mineralogical sources, physical origins, and geophysical applications, and comparison with susceptibility anisotropy. Pure Appl. Geophys., 136: 1-28.

Lee, J.H., Peacor, D.R., Lewis, D. and Wintsch, R.P., 1986 Evidence for syntectonic crystallization for the mudstone to slate transition at Lehigh Gap, Pennsylvania, U.S.A. J. Struct. Geol., 8: 767-- 780 .

Owens, W.H., 1974. Mathematical model studies on factors affecting the magnetic anisotropy of deformed rocks. Tectonophysics, 24: 115-131.

Pfleiderer, S. and Halls, H.C., 1990. Magnetic susceptibility anisotropy of rocks saturated with ferrofluid: a new method to study pore fabric? Phys. Earth Planet. Inter., 65: 158164.

Piper, J.D.A., 1987. Palaeomagnetism and The Continental Crust. Open University Press, Milton Keynes, 434 pp.

Rochette, P., 1987. Magnetic susceptibility of the rock matrix related to magnetic fabric studies. J. Struct. Geol., 9: $1015-1020$.

Rochette, P. and Vialon, P., 1984. Development of planar and linear fabrics in Dauphinois shales and slates (French Alps) studied by magnetic anisotropy and its mineralogical control. J. Struct. Geol., 6: 33-38.

Wintsch, R.P., 1978. A chemical approach to the preferred orientation of mica. Geol. Soc. Am. Bull, 89: 1715-1718.

Wintsch, R.P., Kvale, C.M. and Kisch, H.J., 1991. Open-system, constant-volume development of slaty cleavage, and strain-induced replacement reactions in the Martinsburg Formation, Lehigh Gap, Pennsylvania. Geol. Soc. Am. Bull., 103: 916-927. 